Proyecciones

Vol. 24, No 3, pp. 229-237, December 2005.

Universidad Católica del Norte

Antofagasta - Chile

\title{
FIXED POINTS OF A FAMILY OF EXPONENTIAL MAPS
}

\author{
$E R I C B L A B A C^{*}$ \\ and \\ JUSTIN PETERS \\ Iowa State University, U. S. A.
}

Received : September 2005. Accepted : November 2005

\begin{abstract}
We consider the family of functions $f_{\lambda}(z)=\exp (i \lambda z), \lambda$ real. With the help of MATLAB computations, we show $f_{\lambda}$ has a unique attracting fixed point for several values of $\lambda$. We prove there is no attracting periodic orbit of period $n \geq 2$.
\end{abstract}

Keywords subjclass : 37510

\footnotetext{
${ }^{*}$ Eric Blabac is an MS student in the Mathematics Department at Iowa State University.
} 


\section{Introduction}

In this note we show the existence and uniqueness of an attracting fixed point for the map $f_{\lambda}(z)=\exp (i \lambda z), z \in \mathbf{C}$, for certain (real) values of the parameter $\lambda$. The proofs depend on MATLAB calculations, and as such can be viewed as computer-assisted proofs. By contrast, the exponential map $z \mapsto \exp (z)$ admits no attracting fixed point. ([2], see Remark 12)

We began with a MATLAB program in which one inputs a value $\lambda$, a point $z_{0}$ a positive integer $N$, and a tolerance $\epsilon$. The program outputs $f^{n}\left(z_{0}\right)$, where $n$ is the least integer $k \leq N$ for which

$$
\left|f^{k-1}\left(z_{0}\right)-f^{k}\left(z_{0}\right)\right|<\epsilon
$$

if there is such an $n$. Here $f^{n}(z)$ is the $n$-fold iterate of $f$ at $z$ : thus, $f^{1}(z)=f(z)$, and $f^{n}(z)=f\left(f^{n-1}(z)\right)$ for $n>1$.

We experimented with various values of $\lambda$ and the computations indicated the function $f_{\lambda}$ had a fixed point inside the circle $|z| \leq 1 /|\lambda|$ for $|\lambda| \leq 1.96$ (approximately). However in order to prove the existence of a fixed point, one must use a theorem, which usually involves an invariant domain. Since it was not easy to find such a domain, we took another approach, using the Maximum Modulus Theorem. While the analysis is elementary, the computer-assisted proofs have yielded new results.

Definition 1. A point $z \in \mathbf{C}$ is called an attracting fixed point of a map $f$ if $z$ satisfies $f(z)=z$ and $\left|f^{\prime}(z)\right|<1$.

Suppose that $z_{0}$ is an attracting fixed point of the map $f(z)$. Then for $z$ sufficiently close to $z_{0}$, the iterates $z, f(z), f^{2}(z), \ldots$ converge to $z_{0}$.

Let's begin by stating some facts. Let $f$ be the function $f_{\lambda}$.

Fact 1. If $z_{0}$ is an attracting fixed point of $f$, then $\left|z_{0}\right|<1 /|\lambda|$.

Conversely, any fixed point in the disk $|z|<1 /|\lambda|$ is attracting.

Proof. We have $\left|f^{\prime}\left(z_{0}\right)\right|=\left|i \lambda f\left(z_{0}\right)\right|=|\lambda|\left|z_{0}\right|<1 \Longleftrightarrow\left|z_{0}\right|<1 /|\lambda|$.

Fact 2. For $z \in \mathbf{C}, z=x+i y(x, y$ real $),\left|f^{\prime}(z)\right|<1 \Longleftrightarrow(\operatorname{sgn} \lambda) y>$ $\frac{1}{|\lambda|} \log (|\lambda|)$.

Proof. If $z=x+i y$,

$$
\left|f^{\prime}(z)\right|=|i \lambda \exp (i \lambda z)|=|\lambda| e^{-\lambda y}
$$


The conclusion is an easy exercise.

Fact 3. The region $\mathbf{R}_{\lambda}$ defined by the inequalities

$$
|z|<\frac{1}{|\lambda|}, \quad \operatorname{sgn}(\lambda) y>\frac{1}{|\lambda|} \log (|\lambda|), z=x+i y
$$

is empty if $|\lambda| \geq e$.

Proof. $\frac{1}{\lambda \lambda} \log (|\lambda|)<\operatorname{sgn}(\lambda) y \leq|z|<\frac{1}{\mid \lambda}$, so $\log (|\lambda|)<1$, or $|\lambda|<e$.

Our first goal was to prove the existence of a fixed point inside the circle $|z|=1 /|\lambda|$. We did this using the Maximum Modulus Theorem, or rather a Corollary, called the Minimum Modulus Theorem. Our MATLAB calculations indicated the attracting fixed point should lie in the intersection of $|z| \leq 1 / \lambda$ with the first quadrant (for $\lambda>0$ ), denoted $\mathcal{Q}_{\lambda}$ (or simply $\mathcal{Q}$ if $\lambda$ is fixed), so it was in that region we applied the the Minimum Modulus Principle.

A more conventional approach to the existence of a fixed point using, say, the Brouwer Theorem, requires a having region which is mapped into itself by $f$. But neither $\mathcal{Q}_{\lambda \lambda}$ nor $\mathcal{R}_{\lambda}$ (see Fact 3) is invariant. Say $\lambda>$ $1,0 \in \mathcal{Q}_{\lambda}$ but $1=f_{\lambda}(0) \notin \mathcal{Q}_{\lambda \lambda}$. Also $\frac{i}{\lambda} \in \mathcal{R}_{\lambda}$, but $f_{\lambda}\left(\frac{i}{\lambda}\right)=e^{-1} \notin \mathcal{R}_{\lambda}$ since $\mathcal{R}_{\lambda}$ is disjoint from the real axis if $\lambda>1$. (But see Remark 9.)

Remark 2. It is enough to determine fixed points of $f_{\lambda}$ for $\lambda>0$, for

$$
f_{-\lambda}(\bar{z})=\exp (-i \lambda \bar{z})=\exp (\overline{(i \lambda z)})=\overline{f_{\lambda}(z)},
$$

the last equality resulting from the fact the power series has real coefficients. Also, it is an easy observation that $\overline{z_{0}}$ is an attracting fixed point for $f_{-\lambda}$ iff $z_{0}$ is an attracting fixed point for $f_{\lambda}$.

Notation 1. We use both ' $\exp (\cdot)$ ' and ' $e$ ' ' to denote the exponential function.

\section{Existence and Uniqueness of the Fixed Point}

Theorem 3. Minimum Modulus Principle ([3], p. 313)

Let $U$ be a bounded open set in $\mathbf{C}$, and let $f$ be analytic on $U$ and continuous on the closure $\bar{U}$. Assume that $f$ never vanishes on $\bar{U}$. Then the minimum value of $|f|$ on $\bar{U}$ occurs on the boundary, $\partial U$. 


\section{Theorem 4.}

Let $\lambda \in \mathbf{R}$ be one of the values $0.1,0.2,0.3, \ldots, 1.8,1.9,1.95$, or 1.96 . Let $\mathcal{Q}$ be the intersection of the first quadrant $\Re z \geq 0, \Im z \geq 0$, with the closed disk $|z| \leq 1 / \lambda$. Then there exists a fixed point $z$ in the interior $\mathcal{Q}^{o}$ of $\mathcal{Q}$ for the map $f(z)=\exp (i \lambda z)$.

Proof. Our calculations using MATLAB show that

$$
\left[\min \{z \in \mathcal{Q}:|z-f(z)|\}<10^{-6} .\right.
$$

The idea of the proof is to show $|z-f(z)|$ is bounded below along the boundary of $\mathcal{Q}$ by some constant which is greater than $10^{-6}$. Theorem would then assert the existence of a fixed point for $f$ in $\mathcal{Q}$. Writing $z=x+i y$, for $x, y \in \mathbf{R}$ we have

$$
\begin{array}{r}
|f(z)-z|^{2}=|\exp (i \lambda z)-z|^{2} \\
=\left|e^{-\lambda y} e^{i \lambda x}-(x+i y)\right|^{2} \\
=\left|e^{-\lambda y}(\cos (\lambda x)+i \sin (\lambda x))-(x+i y)\right|^{2} \\
=e^{-2 \lambda y}+x^{2}+y^{2}-2 e^{-\lambda y}(x \cos (\lambda x)+y \sin (\lambda x)) .
\end{array}
$$

Denote the right hand side of the above by $g(x, y)$. We now have to check the values of $g(x, y)$ along the boundaries of $\mathcal{Q}$. We will start with $y=0$. Then for $0 \leq x \leq 1 / \lambda$

$$
g(x, 0)=x^{2}+1-2 x \cos (\lambda x) .
$$

Thus

$$
g(x, 0)=(x-1)^{2}+2 x(1-\cos (\lambda x)) .
$$

Since $0<\lambda<e$ we have $g(x, 0) \geq\left(1-e^{-1}\right)^{2}$ on $0 \leq x \leq e^{-1}$ and by $2 e^{-1}\left(1-\cos \left(\frac{\lambda}{e}\right)\right) \geq 2 e^{-1}(1-\cos (1))$ on $e^{-1} \leq x \leq \lambda^{-1}$.

Next we check the boundary $x=0,0 \leq y \leq 1 / \lambda$ :

$$
g(0, y)=y^{2}+e^{-2 \lambda y} \geq e^{-2 \lambda y} \geq \frac{1}{e^{2}} .
$$

Finally we need to check the boundary on the quarter circle; it is convenient to convert to polar coordinates

$$
x=(1 / \lambda) \cos \theta, \quad y=(1 / \lambda) \sin \theta, \text { for } 0 \leq \theta \leq \pi / 2 .
$$


Then,

$$
\begin{aligned}
& g\left(\frac{1}{\lambda} \cos \theta, \frac{1}{\lambda} \sin \theta\right)= \\
& \left(\frac{1}{\lambda}\right)^{2}+e^{-2 \sin \theta}-e^{-\sin \theta}\left(\frac{2}{\lambda} \cos \theta \cos (\cos \theta)-\frac{2}{\lambda} \sin \theta \sin (\cos \theta)\right)
\end{aligned}
$$

and using the identity $\cos (\alpha \pm \beta)=\cos \alpha \cos \beta \mp \sin \alpha \sin \beta$ we see that

$$
\begin{array}{r}
g\left(\frac{1}{\lambda} \cos \theta, \frac{1}{\lambda} \sin \theta\right)=\left(\frac{1}{\lambda}\right)^{2}+e^{-2 \sin \theta}-e^{-\sin \theta}\left(\frac{2}{\lambda} \cos (\theta-\cos \theta)\right) \\
=\left(\lambda^{-1}-e^{-\sin \theta}\right)^{2}+\frac{2}{\lambda} e^{-\sin \theta}(1-\cos (\theta-\cos \theta)) .
\end{array}
$$

Since both $\left(\lambda^{-1}-e^{-\sin \theta}\right)^{2}$ and $\frac{2}{\lambda} e^{-\sin \theta}(1-\cos (\theta-\cos \theta))$ are nonnegative, we must see where both terms are zero: the second expression is zero only when $\cos \theta=\theta$. Call this $\theta_{0}$; so $\theta_{0}$ is approximately .739085. Putting $\theta=\theta_{0}$ into the first term and setting it to zero yields $\lambda=\lambda_{0}:=e^{\sqrt{1-\theta_{0}^{2}}}$, or approximately 1.96131 .

From this we can conclude: suppose $\lambda$ is in the interval $0<\lambda \leq 1.96$. Then for $0 \leq \theta \leq .741$,

$$
\lambda^{-1}-e^{-\sin \theta} \geq 1.96^{-1}-e^{-\sin .741}>10^{-3} .
$$

And for $.741 \leq \theta \leq \frac{\pi}{2}$,

$$
\begin{aligned}
& \frac{2}{\lambda} e^{-\sin \theta}(1-\cos (\theta-\cos \theta)) \\
& \geq \frac{2}{1.96} e^{-\sin .741}(1-\cos (.741-\cos .741))>(2.67) 10^{-6}
\end{aligned}
$$

We conclude that $|g(x, y)|^{\frac{1}{2}}>10^{-3}$ on the boundary of $\mathcal{Q}$. Since

$$
\min _{z \in \mathcal{Q}}\{|z-f(z)|\}=\min _{(x, y) \in \mathcal{Q}}\left\{g(x, y)^{\frac{1}{2}}\right\}<10^{-6}
$$

for any $\lambda$ in the Table, it follows from the Minimun Modulus Principle that the function $f(z)=\exp (i \lambda z)$ has a fixed point.

Corollary 5. From Fact 1, any fixed point for $f$ inside the circle $|z|<\frac{1}{\lambda}$ is attracting, so that Theorem 4 establishes the existence of an attracting fixed point in $\mathcal{Q}^{\circ}$. By Fact 2, the fixed point lies in the intersection $\mathcal{Q}^{\circ} \cap \mathbf{R}$.

Below are the approximate fixed points for different values of $\lambda$. For each $\lambda$ the program terminated when $|f(z)-z|<10^{-8}$.

Table of Fixed Points 


$\begin{array}{cccc}\lambda & \text { Fixed Point } & \lambda & \text { Fixed point } \\ .1 & 0.9854986+0.0974364 i & 1.1 & 0.5463106+0.3745068 i \\ .2 & 0.9470891+0.1815722 i & 1.2 & 0.5191342+0.3729401 i \\ .3 & 0.8954147+0.2464877 i & 1.3 & 0.4945130+0.3703950 i \\ .4 & 0.8396697+0.2931240 i & 1.4 & 0.4721254+0.3671575 i \\ .5 & 0.7852571+0.3251993 i & 1.5 & 0.4516947+0.3634355 i \\ .6 & 0.7346292+0.3465471 i & 1.6 & 0.4329840+0.3593815 i \\ .7 & 0.6885773+0.3602367 i & 1.7 & 0.4157904+0.3551080 i \\ .8 & 0.6470987+0.3685124 i & 1.8 & 0.3999401+0.3506985 i \\ .9 & 0.6098583+0.3729619 i & 1.9 & 0.3852840+0.3462151 i \\ 1.0 & 0.5764127+0.3746990 i & 1.95 & 0.3783627+0.3439606 i \\ & & 1.96 & 0.3770090+0.3435093 i\end{array}$

One might infer from our results that $f_{\lambda}$ has a attracting fixed point for all values of $\lambda, 0<\lambda<\lambda_{0}$. But our method of proof can only be applied to finitely many $\lambda$.

Remark 6. For $\theta_{0}, \lambda_{0}$ as in the proof of the theorem, the proof shows that the function $f_{0}(z)=\exp \left(i \lambda_{0} z\right)$ has a fixed point at $z=z_{0}:=\lambda_{0}^{-1} e^{i \theta_{0}}$. Since $z_{0}$ lies on the circle $|z|=1 / \lambda_{0}$, it follows $\left|f_{0}^{\prime}\left(z_{0}\right)\right|=1$. Such a point is called a nonhyperbolic, or neutral fixed point.

Remark 7. It is possible to express the fixed point $z$ of $f_{\lambda}$ as an analytic function of $\lambda$. Solving $z=f_{\lambda}(z)$ for $\lambda$ yields $\lambda=-i \log (z) / z$. The inverse function is given by $z=g(\lambda):=i W(-i \lambda) / \lambda$ where $W$ is the Lambert $W$ function, or the principal branch of the inverse of $w \rightarrow w e^{w}$. Since the values of $W$ are not easy to calculate, this does not simplify the question of deciding when the fixed point $z=g(\lambda)$ is attracting, i.e., when it satisfies $|g(\lambda)|<\frac{1}{\lambda} .(\mathrm{Cf}[1]$.

Our MATLAB computation indicates the fixed point is unique. That is indeed the case, as we now prove.

Theorem 8. Let $\lambda$ be one of the values in the Table. Then the map $f(z)=\exp (i \lambda z)$ has a unique attracting fixed point.

\section{Proof.}

Let $\mathbf{R}$ be the region in $\mathcal{C}$ determined by the two inequalities $x^{2}+y^{2}<$ $1 / \lambda^{2}$ and $y>\frac{1}{\lambda} \log (\lambda)$. The region $\mathbf{R}$ is convex, and by Facts 1,2 , and 3 it is nonempty, and contains all attracting fixed points of the map $f$. 
Suppose now that $z_{1}, z_{2}$ are two distinct fixed points of $f$. Then $z_{1}, z_{2}$ lie in $\mathbf{R}$, and if $\mathcal{C}$ is a contour joining $z_{1}$ and $z_{2}$,

$$
\begin{aligned}
\left|z_{1}-z_{2}\right| & =\left|f\left(z_{1}\right)-f\left(z_{2}\right)\right|=\left|\int_{\mathcal{C}} f \prime(z) d x\right| f \prime(z) d x \mid \\
\leq & \int_{\mathcal{C}}|f \prime(z)| d|z| \leq \max _{z \in \mathcal{C}}\left\{\left|f^{\prime}(z)\right|\right\} \operatorname{length}(\mathcal{C}) .
\end{aligned}
$$

If $\mathcal{C}$ is the straight line contour joining $z_{1}$ and $z_{2}$, then $\mathcal{C} \subset \mathbf{R}$ so that $|f \prime(z)|<1$ for $z \in \mathcal{C}$, and since $\mathcal{C}$ is compact, $\max _{z \in \mathcal{C}}\{|f \prime(z)|\}<1$. Since length $(\mathcal{C})=\left|z_{1}-z_{2}\right|$ the calculation above implies $\left|z_{1}-z_{2}\right|<\left|z_{1}-z_{2}\right|$, which is absurd. Thus the fixed point is unique.

Remark 9. An alternative, more conventional approach to the existence of a fixed point may be possible using a standard fixed point theorem, such as the Brouwer Theorem. Assume that $z_{0}$ satisfies $\left|f\left(z_{0}\right)-z_{0}\right|<10^{-6}$, and $\left|f^{\prime}\left(z_{0}\right)\right|<1$.Let $\epsilon=1-\left|f^{\prime}\left(z_{0}\right)\right|$. There is a $\delta>0$ such that $\left|f^{\prime}(z)\right|<1-\epsilon / 2$ for $\left|z-z_{0}\right|<\delta$. So for $\left|z-z_{0}\right| \leq \delta$,

$$
\begin{array}{r}
\left|f(z)-z_{0}\right| \leq\left|f(z)-f\left(z_{0}\right)\right|+\left|f\left(z_{0}\right)-z_{0}\right| \\
<\max \left\{\left|f^{\prime}(z)\right|: z \in \mathcal{C}\right\} \delta+10^{-6} \\
<(1-\epsilon / 2) \delta+10^{-6} \\
<\delta
\end{array}
$$

(where $\mathcal{C}$ is the line segment joining $z$ and $z_{0}$ ) is valid as long as $10^{-6}<\frac{\epsilon}{2} \delta$. A tolerance finer than $10^{-6}$ may be required. We have not carried out these calculations. However, we do not see how the critical value $\lambda_{0}$ could be obtained through this approach.

\section{Attracting Orbits}

If $z_{0}, z_{1}, \ldots, z_{n-1}$ is a set of points satisfying

\section{Remark 1.}

$$
z_{1}=f\left(z_{0}\right), z_{2}=f\left(z_{1}\right), \ldots, z_{0}=f\left(z_{n-1}\right)
$$

then $z_{0}, z_{1}, \ldots, z_{n-1}$ is called an orbit of period $n$.

Definition 10. Let $z_{0}, z_{1}, \ldots, z_{n-1}$ be an orbit of period $n$. It is said to be an attracting orbit if $\left|f^{\prime}\left(z_{k}\right)\right|<1,0 \leq k \leq n-1$.

Let $f(z)=\exp (i \lambda z), \lambda>0$, and $z_{0}, z_{1}, \ldots, z_{n-1}$ a period $n$ orbit, and assume the orbit is attracting. Observe 


$$
\left|f^{\prime}\left(z_{0}\right)\right|=\left|i \lambda \exp \left(i \lambda z_{0}\right)\right|=\lambda\left|z_{1}\right|<1 \text {. }
$$

Similarly, $z_{2}, \ldots, z_{n-1}, z_{0}$ lie in the circle $|z|<1 / \lambda$.

Furthermore, it follows from Fact 2 that $z_{k}$ satisfy $y_{k}>\frac{1}{\lambda} \log (\lambda)$, where $z_{k}=x_{k}+i y_{k}$. Thus, $z_{0}, z_{1}, \ldots, z_{n-1}$ lie in the region $\mathbf{R}$ in the complex plane determined by the two inequalities $x^{2}+y^{2}<1 / \lambda^{2}$ and $y>\frac{1}{\lambda} \log (\lambda)$.

Theorem 11. Let $0 \neq \lambda \in \mathbf{R}$. Then the map $f(z)=\exp (i \lambda z)$ does not have any attracting periodic orbit of period $n$, for $n \geq 2$.

Proof. As noted above (cf Remark 2) it is enough to prove the assertion for $\lambda>0$. The proof is in the spirit of the uniqueness proof (Theorem 8 ).

Note our definition of attracting orbit is stronger than the standard definition ([2]): $\left|\left(f^{n}\right)^{\prime}\left(z_{0}\right)\right|<1$, or equivalently that $\left|f^{\prime}\left(z_{0}\right) f^{\prime}\left(z_{1}\right) \cdots f^{\prime}\left(z_{n-1}\right)\right|<$ 1.

Remark 12. Recall that the Julia set of a map $f$ is the closure of the repelling periodic points. It's interesting to note the difference between the maps $f_{\lambda}$ and the exponential map, $z \rightarrow e^{z}$. For the exponential map, it is shown in [2] that the Julia set is all of $\mathbf{C}$. But for the maps $f_{\lambda}$ (at least for the values of $\lambda$ in the table), the Julia set is a proper subset: indeed, there is an open neighborhood $U$ of the attracting fixed point, which is invariant under $f_{\lambda}$, not containing any other periodic points. Thus, the Julia set of $f_{\lambda}$ is a proper subset of $\mathbf{C}$.

Of course each $f_{\lambda}$ has a Julia set which is unbounded, and hence $f_{\lambda}$ has infinitely many repelling periodic points.

\section{References}

[1] Borwein, Jonathan M, and Corless, Robert M., Emerging Tools for Experimental Mathematics, Amer. Math. Monthly 106, No. 10, pp. 899 909, (1999).

[2] Devaney, Robert L., An Introduction to Chaotic Dynamical Systems, Addison-Wesley, (1989). 
[3] Rubenfeld, Lester A., A First Course in Applied Complex Variables, John Wiley \& Sons, (1985).

Eric M. Blabac

Department of Mathematics

Iowa State University

Ames, IA 50011

U. S. A.

e-mail : ebikeman@iastate.edu

and

Justin R. Peters

Department of Mathematics

Iowa State University

Ames, IA 50011

e-mail : peters@iastate.edu 